\title{
Levels of lactoferrin, lysozyme and albumin in the tear film of keratoconus patients and their correlations with important parameters of the disease
}

\author{
Miruna Gabriela Burcel ${ }^{1 *}$, Mihaela Constantin ${ }^{1}$, Gabriela Ionita ${ }^{2}$, Dana \\ Dascalescu ${ }^{3}$, Catalina Ionescu ${ }^{4}$, Dan Stanila ${ }^{5}$, Vasile Potop ${ }^{6}$, Valeria Coviltir ${ }^{6}$ \\ 1. Oftaclinic Clinic, Bucharest, Romania \\ 2. Institute of Physical Chemistry "Ilie Murgulescu", Bucharest, Romania \\ 3. Faculty of Dental Medicine, "Carol Davila” University of Medicine \\ and Pharmacy, Bucharest, Romania \\ 4. CMDTAMP Bucharest, Romania \\ 5. Faculty of Medicine, Lucian Blaga University, Sibiu, Romania \\ 6. Clinical Hospital of Ophthalmologic Emergencies, Bucharest, Romania
}

\begin{abstract}
Background: Despite the fact that keratoconus has been tipically defined as a noninflammatory condition, recent research has promoted the role of inflammatory factors and protein changes of tear film in disease progression. Aims: to determine the level of serum albumin, lactoferrin and lyzozyme in tears of keratoconic patients and their correlations with corneal biomechanical properties. Subjects and methods: 16 eyes of keratoconus patients and 14 eyes of control cases were enrolled in an observational prospective study. We performed a complete ophthalmological examination on all participants. In order to determine the concentration of tear film proteins, a minimum of 20 microlitres of tears from the lower conjunctival fornix were collected from each subject and measured by enzyme-linked immunosorbent assay (ELISA) analysis. Results: The level of lactoferrin measured in the tear film was significantly decreased in the keratoconus group compared to the normal subjects in all cases $(\mathrm{p}<0.05)$. We also found an increased level of lyzozyme and albumin in the keratoconus patients when compared to the controls, only the lyzozyme beeing statistically significant. In the keratoconus group, the correlations between proteins and important parameters such as keratometry, pachymetry and corneal biomechanics were statistically relevant in our study. Conclusions: We can state that the protein composition of tears is modified in keratoconus by increased levels of protein with inflammatory properties such as albumin or by decreased levels of protein with anti-inflammatory properties such as lactoferrin.
\end{abstract}

Keywords: keratoconus, lyzozyme, albumin, lactoferrin

Received: $8^{\text {th }}$ September 2019; Accepted: 19 $9^{\text {th }}$ February 2020; Published: $2^{\text {nd }}$ March 2020

*Corresponding author: Miruna Gabriela Burcel, Oftaclinic Clinic, Bucharest, Romania.

E-mail: cristea.miruna@yahoo.com 


\section{Introduction}

Keratoconus is a common disease of the cornea, characterized by progressive thinning of the corneal stroma leading to significant visual impairment and corneal scarring (1). Its ethiology is not completely understood untill now, both genetic and environmental factors being involved. It is recognized that an environmental factor acts as a trigger of the disease in the people that are genetically predisposed. Keratoconus may be isolated, associated with various clinical conditions such as atopy, ocular allergies, or even part of a syndrome: Down, Ehlers Danlos, Marfan, Noonan syndromes (2-4). Besides atopy and ocular allergies, other environmental factors, which have been described, are eye rubbing and sun exposure. These two, may cause oxidative damage to keratoconic corneas due to a reduced amount of corneal enzymes such as aldehyde dehydrogenase 3 (ALDH3) and superoxid dismutase to process reactive oxygen species (ROS), which determine a degradation process with corneal thinning and loss of vision $(3,5)$.

Despite the fact that keratoconus has been tipically defined as a noninflammatory condition, recent research has promoted the role of inflammatory factors and protein changes of tear film in disease progression (6). Some authors found that stromal thinning appears due to a combination of increased activity of proteases and other catabolic enzymes or by decreased proteinase inhibitors (7). Also Lema et al reported an overexpression of proinflammatory cytokines such as interleukin 6 (IL-6) and tumor necrosis factor alpha (TNF- $\alpha$ ), together with matrix metalloproteinase (MMP)-9 in the tears of keratoconic patients (8). All of these generate alterations of extracellular matrix and collagen fibrils that lead to changes in corneal biomechanics.

Besides proteases and proinflammatory cytokines, the tear fluid of patients with keratoconus contains other biomarkers such as serum albu- min, lyzozyme, lactoferrin, lipocalin, which constitute the most abundant proteins in the tear fluid (9). A keratoconic cornea presents, beside thinning of the stroma, other ultrastuctural changes that can modify corneal strentgh. These changes are thinning of the epithelium and breaks in Bowman's layer, which appear due to ocular itching and rubbing thus provoking chronic epithelial lesions $(7,10)$. A few recent studies have examined the changes to the major tear proteins in keratoconus $(6,11)$. Taking into account all these, the present study evaluates the level of serum albumin, lactoferrin and lyzozyme in tears of keratoconic patients and their correlations with corneal biomechanical properties.

\section{Materials and Methods}

This study was a prospective, case-controlled one which included 16 eyes of 16 keratoconus (KC) patients (11 males and 5 females) and 14 eyes of 14 control patients ( 9 males and 5 females). In the set of keratoconus the mean age was $26.13 \pm 8.79$ (range 14-44), while the controls had a mean age of $26.57 \pm 9.79$ with ranging between 11 and 37. Study participants were recruited from Oftaclinic Ophthalmology Clinic, Bucharest, Romania between January 2017 and August 2017. The University of Medicine and Pharmacy "Carol Davila" Bucharest approved the protocol (no.130/2017) and an informed consent was obtained from all patients and control subjects before the enrollment in the study. Inclusion criteria were as follows: 1) patients with $\mathrm{KC}$ confirmed using Amsler Krumreich classification based on biomicroscopy, keratometry, refraction error and central corneal thickness $(12)$; 2) participants in the control group without modifications on corneal topography maps.

Exclusion criteria included: history of ocular surgery, ocular or systemic allergy, presence of local or systemic inflammation, or treatment 
with local or systemic anti-inflammatory drugs, dry eye disease.

Corneal topography was performed using Topcon device, the biomechanical characteristics were recorded using Ocular Response Analyzer (Reichert, Depew, NJ) and corneal thickness (CCT) was recorded using ultrasonic pachymeter for all patients.

Tear collection was performed carefully without topical anesthesia. We used $50 \mu \mathrm{l}$ capillary tubes to collect a minimum of $20 \mu \mathrm{l}$ of tear volume from the inferior conjunctival cul-du-sac (avoiding touching the conjuntival surface) by capillary attraction and then transferred in Eppendorf tubes. After that, the tear samples were stored at $-80{ }^{\circ} \mathrm{C}$ within 1 hour without centrifugation until analysis.

Tear analysis for lactoferrin, lysozyme and serum albumin was made using Human LTF (Lactoferrin) ELISA kit, Human LZM (Lysozyme) ELISA kit and Human AAA ( Anti-Albumin Antibody) ELISA kit (MyBioSource). To prewet the plate, $200 \mathrm{ml}$ wash buffer was used and prepared for incubating on the shake plate. Samples, standards and controls were stored in microtitre wells, after the specific antibodies to lactoferrin, lysozyme or albumin were added. Consecutively, biotinylated secondary antibody and streptavidin-peroxidase conjugate were added to the wells. Then a substrate was added which reacted with the streptavidin conjugate and the solution that resulted was read at $450 \mathrm{~nm}$ using the microplate reader ZENYTH 3100 (Anthos Labtec Instruments GmbH, Salzburg-Austria) and CURVE EXPERT software version 1.4, provided in the kit for each individual analyte.

\section{Statistical analysis}

Statistical analysis was performed using the statistical software SPSS statistics, version 20. The Shapiro-Wilk test was used to check for a normal distribution of quantitative data, appropriate for small sample sizes of fewer than 50 participants.
An independent sample t-test was performed to analyze the difference in outcomes between the two groups, while the Mann-Whitney test was performed when data were not distributed normally. The correlations between the variables were investigated by determining the $r$ Pearson correlation index. Continuous variables are presented as mean+/-standard deviation. A $\mathrm{p}$ value $<0.05$ was considered statistically significant.

\section{Results}

Our results showed no statistically significant age or sex-related differences between $\mathrm{KC}$ patients and control subjects. Keratoconic patients (11 men and 5 women) presented a mean age of $26.13 \pm 8.79$ years and control individuals (9 men and 5 women) presented a mean age of 26.57 \pm 9.79 years. Patients' distribution according to Amsler Krumeich classification was as follows: stage $1-3$ eyes (18.8\%), stage $2-8$ eyes $(26,7 \%)$, stage $3-1$ eyes $(3.3 \%)$, stage $4-4$ eyes $(13.3 \%)$.

The results from clinical records are reported in Table 1. They showed statistically significant changes in $\mathrm{KC}$ patients versus control patients. Patients with $\mathrm{KC}$ present higher corneal values, lower corneal thickness and biomechanical parameters than control group.

Tear serum albumin, lactoferrin and lyzozyme concentration in keratoconic patients and control subjects are shown in Table 2. KC patients had a significantly lower level of lactoferrin $(p=0.001)$ and a higher level of lyzozyme $(p=0.012)$ when compared to the control group. No statistically significant serum albumin level difference was detected between keratoconus and control groups.

Furher on, we carried out some correlations between the protein level in tears and highest corneal value ( $\mathrm{K} \max$ ) and central corneal thickness. A significant negative correlation was found with lactoferrin $(\mathrm{r}=-0.691, \mathrm{p}=0.003)$ and 
Table 1. Descriptive statistics for clinical parameters in keratoconus versus control group

\begin{tabular}{lccc}
\hline Variables & Keratoconus $(\mathbf{n}=\mathbf{1 6})$ & Control $(\mathbf{n}=\mathbf{1 4})$ & P value \\
\hline K max, diopters & $51.89 \pm 3.94$ & $44.14 \pm 1.46$ & $0.0001^{*}$ \\
\hline K min, diopters & $47.33 \pm 4.53$ & $43.35 \pm 1.58$ & $0.004^{*}$ \\
\hline CH & $8.76 \pm 1.16$ & $10.95 \pm 2.43$ & $0.001^{*}$ \\
\hline CRF & $7.31 \pm 1.35$ & $9.94 \pm 2.42$ & $0.001^{*}$ \\
\hline KMI & $0.275 \pm 0.25$ & $0.581 \pm 0.43$ & $0.011^{*}$ \\
\hline CCT $(\mu \mathrm{m})$ & $449.88 \pm 43.77$ & $536.57 \pm 18.74$ & $0.0001^{*}$ \\
\hline
\end{tabular}

Results are resumed (mean $\pm \mathrm{SD}$ ) for $\mathrm{K}$ max, $\mathrm{K}$ min (corneal values on highest respective lowest refractive meridian), corneal histerezis $(\mathrm{CH})$, corneal resistance factor (CRF), keratoconus match index (KMI) and pachymetry (corneal central thickness-CCT). ${ }^{*}$ statistically significant

Table 2 Levels of serum albumin, lactoferrin and lyzozyme in tears

\begin{tabular}{lccc}
\hline Variables & Keratoconus $(\mathbf{n}=\mathbf{1 6})$ & Control $(\mathbf{n}=\mathbf{1 4})$ & P value \\
\hline serum albumin $(\mu \mathrm{g} / \mathrm{ml})$ & $7.49 \pm 5.06$ & $4.35 \pm 2.43$ & 0.130 \\
\hline lactoferrin $(\mathrm{mg} / \mathrm{ml})$ & $0.20 \pm 0.21$ & $1.06 \pm 1.17$ & $0.001^{*}$ \\
\hline lysozyme $(\mathrm{mg} / \mathrm{ml})$ & $2.85 \pm 2.08$ & $1.63 \pm 0.3$ & $0.012^{*}$ \\
\hline
\end{tabular}

Results are presented as mean \pm standard deviation; * statistically significant

a significant positive correlation with lyzozyme $(\mathrm{r}=0.671, \mathrm{p}=0.004)$ when compared to Kmax (Figure1). Also, we obtained no significant lower correlation $(\mathrm{r}=0.147)$ between Kmax recorders and albumin level in keratoconus subjects. The correlations of keratomery with lactoferrin and lyzozyme are shown in Figure 1.

As for the central corneal thickness in keratoconus patients we found a strong, positive, statistically significant correlation with lactoferrin $(r=0.641, p=0.007)$. We also found a weak correlation between pachimetry and lyzozyme $(\mathrm{r}=-0.309, \mathrm{p}=0.244)$, but without statistic significance. There was no significant correlation $(r=0.068, p=0.803)$ between pachymetry and serum albumin.

Taking into account that corneal biomechanics are altered in a keratoconic cornea, we examined the correlation between corneal hysteresis $(\mathrm{CH})$ and corneal resistance factor (CRF) and tear level of proteins. A significant positive correlation was found with lactoferrin $(\mathrm{r}=0.837, \mathrm{p}=0.0001)$ while with lyzozyme we found a negative one $(r=-0.571, p=0.021)$ when compared to corneal hysteresis. We found a weak correlation between $\mathrm{CH}$ and serum albumin, without statistical significance $(\mathrm{r}=-0.386, \mathrm{p}=0.140)$. As for the corneal resistance factor we found a significant positive correlation with lactoferin $(\mathrm{r}=0.779, \mathrm{p}=0.0001)$ and a negative significant correlation with lyzozyme $(\mathrm{r}=-0.670, \mathrm{p}=0.005)$. There was no correlation between CRF and serum albumin ( $\mathrm{r}=-0.038$, $\mathrm{p}=0.888$ ). Correlation analyses with statistical significance were illustrated in Figure 2.

\section{Disscusions}

Despite extensive studies of keratoconus in recent years, the ethiology remains unknown so in this study, we performed a proteomic analysis of the eye with keratoconus patients and control subjects. Our results show altered tear protein profile in the $\mathrm{KC}$ subjects. We found decreased expression of lactoferrin and increased level of lyzozim and serum albumin in keratoconic subjects compared with control subjects. Therefore, we searched some relevant studies in the literature which analyze proteins levels in keratoconic patients in order to compare our results. 


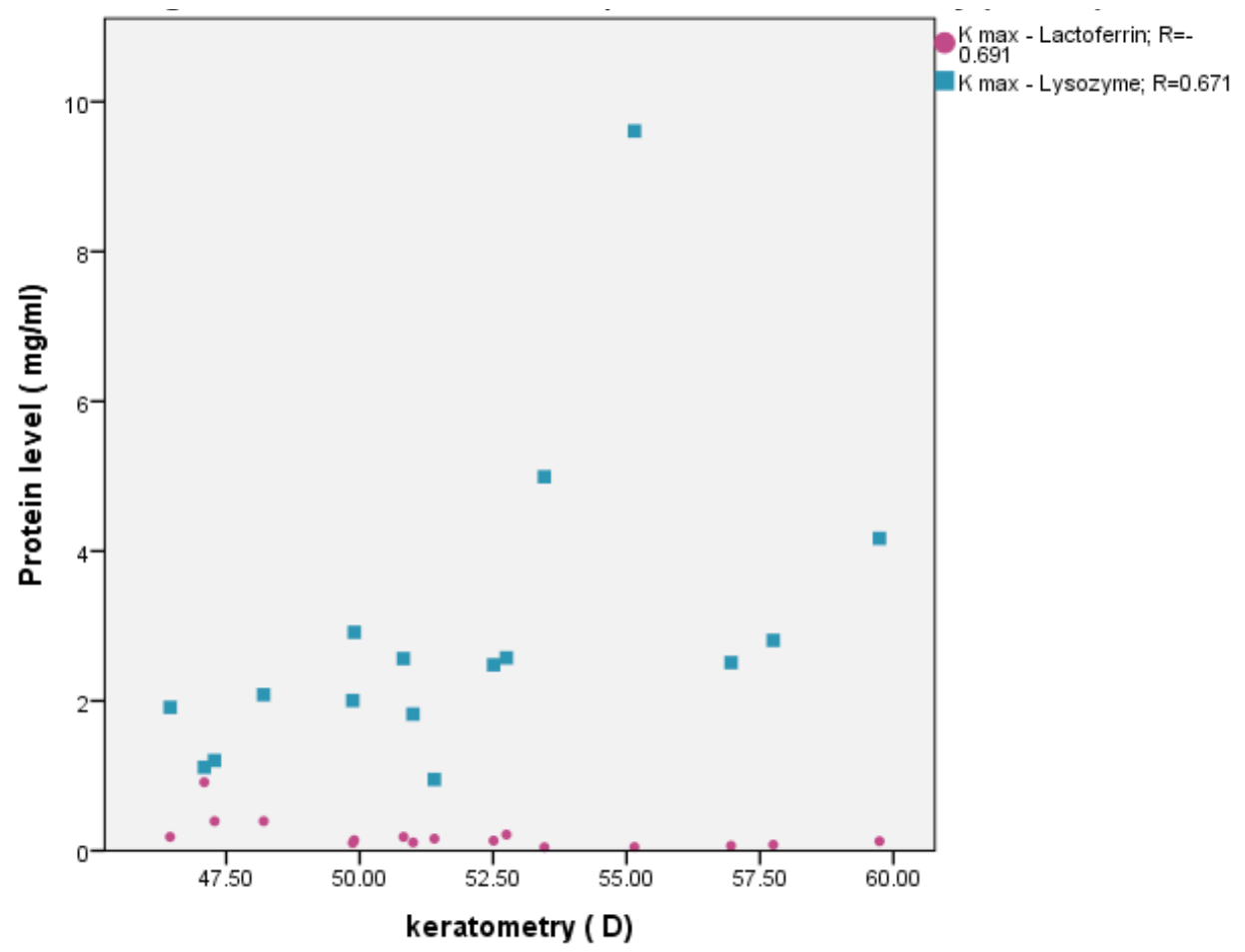

Fig.1. Correlation between tear protein and keratometry (K max)

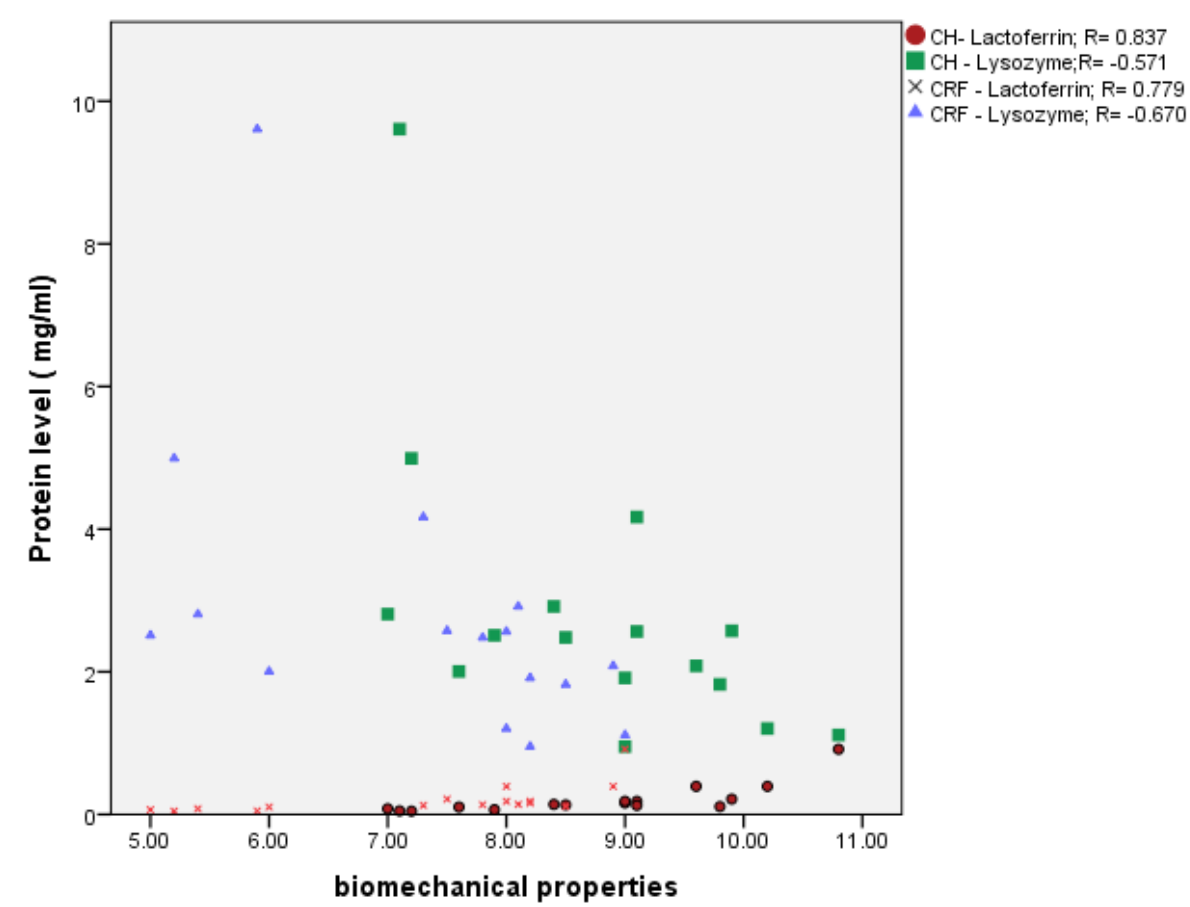

Fig.2. Correlation between tear protein and biomechanical properties (CH, CRF) 
Lactoferrin is a protein that binds iron and represents about $25 \%$ by weight of the total tear proteins (about $2.2 \mathrm{mg} / \mathrm{ml}$ ) (13). However there are studies that reported a variable level of lactoferrin in normal human tears ranging from 0.63 $\mathrm{mg} / \mathrm{ml}$ to $1.49 \mathrm{mg} / \mathrm{ml}$, with an average of 1.42 $\mathrm{mg} / \mathrm{ml}$ (13). Previous studies have demonstrated that levels of lactoferrin are lower in Sicca keratoconjunctivitis, vernal or giant papillary conjunctivitis, and postoperative cataract surgery $(11,13)$. Our study revealed a concentration of lactoferrin of $0.20 \pm 0.21 \mathrm{mg} / \mathrm{ml}$ in the tears of keratoconus patients and of $1.06 \pm 1.17 \mathrm{mg} / \mathrm{ml}$ in the tears of controls. This result was in accordance to Willcox et al. who found a concentration of $0.67 \pm 0.28 \mathrm{mg} / \mathrm{ml}$ in keratoconic patients versus $1.13 \pm 0.29 \mathrm{mg} / \mathrm{ml}$ in healthy people (11). Lactoferrin has an antioxidant, antibacterial (synergism with lysozyme) and anti-inflammatory activity (stimulation and/or repression of cytokines' delivery). Being characterized by anti-inflammatory properties, it inhibits the proinflammatory effects of the hydroxyl radicals (13). Also results from a number of studies have assessed that lactoferrin inhibits the production of IL- 6 , TNF- $\alpha$, interleukin beta (IL-a- $\beta$ ) and interleukin 8 (IL-8). Therefore the increased expression of proinflammatory cytokines such as IL-6, TNF- $\alpha$ in the tears of patients with KC may be explained by the decreased level of lactoferrin (14). Being a protein that determines depletion of free iron, the decreased levels of lactoferrin in keratoconic corneas induce the deposition of free iron on corneal endothelium, which is called Fleischer's ring sign (11).

Lysozyme is a glycolytic enzyme produced by lysosomes, which represent $20-40 \%$ of the total normal tear proteins. Being a protein with antibacterial function, it destroys bacteria by breaking down the peptidoglycan within the bacterial cell walls. Lysozyme interacts with lactoferrin, which decreases the overall negative charge on the bacterial surface, allowing greater acces- sibility of lysozyme to the underlying peptidoglycans. Studies have reported a normal tear lysozyme level range between 0.6 and $2.6 \mathrm{mg} / \mathrm{ml}$ (15). We found an increased expression of lysozyme in keratoconus of $2.85 \pm 2.08 \mathrm{mg} / \mathrm{ml}$ when compared to healthy people were we found a level of $1.63 \pm 0.3 \mathrm{mg} / \mathrm{ml}$. Gorskova et al studied in 1998 the local immunity on 146 eyes from 81 patients with keratoconus at different stages of the disease and published a series of results, suggesting increased concentrations of secretory imunoglobulin $\mathrm{A}(\operatorname{Ig} \mathrm{A})$, immune complexes, C3 component of the complement, transferrin, $\alpha 1$-antitrypsin and lysozyme in the tears of keratoconus patients (16). These findings were similar to ours, being reflected in classifying keratoconus as an inflammatory disease.

Serum albumin represents a minor component of human tears, but it can rise markedly with conjunctival stimulation (15). When serum albumin is present in tears, this indicates the failure of blood-ocular barrier in vessels of the conjunctiva (6). Our study results show an increased expression in albumin in tears from keratoconic patients versus controls, but without statistical significance. The increased level of albumin in keratoconus patients' tears may be indicative of albumin exudation from blood vessels. Balasubramian et al observed a decresed level in serum albumin concentration in keratoconus patients without being statistically significant, a situation that contradicts our results (11). It is also known that an increased concentration of serum albumin in tears in associated with the dry eye syndrome $(11,17)$. Taking into account that it is now known that inflammation is one of the key pathologies in keratoconus, it is not unexpected that some of the biomarkers such as lactoferrin and albumin are similar between keratoconus and dry eye.

Moreover, we investigated if there is any correlation between keratoconus severity and the tear proteins and found a positive correlation 
between lysozyme and maximum keratometry and a negative one between lactoferrin and keratometry, indicating that a more severe stage of the disease is associated with a higher level of lysozyme and a lower level of lactoferrin. Given the fact that the decreased level of lactoferrin in keratoconus may explain the increased expression of proinflammatory cytokines we can state that the higher the local inflammation and the reduced antiinflammatory capacity in the tears of KC patients, the more important the severity of the disease. However, the increased serum albumin in KC tears, which may be another sign of local inflammation, did not correlate with the keratometry readings. Further studies are necessary in order to determine the effect of keratoconus on tear film albumin. Balasubramanian et al also investigated the correlation between keratoconus stages and the tear proteins and proved a negative correlation between lactoferrin and keratometry and no significant correlation between albumin and keratometry, which are in accordance with our study (11).

Taking into account that inflammatory cytokines and proteolytic enzymes, which interact with tear proteins generating a corneal thinning and thus interfere in the mechanical properties of the cornea, we investigated if there is any relationship between tear film proteins and corneal biomechanical properties. To our knowledge, this is the first study which has investigated the interactions between corneal biomechanics and tear proteins. A significant positive correlation was found with lactoferrin when compared to corneal hysteresis and the corneal resistance factor, respectively a significant negative one with the lysozyme. There were no significant correlations between corneal biomechanics and the tear concentration of albumin. The positive correlation between lactoferrin and corneal hysteresis and corneal resistance factor means that a low level of LTF is associated with low corneal biome- chanical stability. Since biomechanical stability is dependent on organization of structural components within the cornea, the microstructural alterations encountered in keratoconus lead to abnormal biomechanical response when compared to normal corneas (18). The microstructural alterations involve tissue degradation due to the overexpression of proinflammatory cytokines and matrix metalloproteinases, which is related to the degree of decreased level of lactoferrin encountered in keratoconus. Furthermore, we can state the lower the level of antiinflammatory molecules, the more important the alteration of biomechanical stability.

An important limitation of our study is the relatively small number of participants. The low number of included subjects decreases the strength with which we may state our conclusions. We based our statements on the fact that similar proteomic studies have used similar sample sizes $(12,15)$.

\section{Conclusions}

Although there are studies that examined the tear film proteins of keratoconic corneas, this study has quantified lactoferrin, lysozyme and serum albumin and for the first time it has correlated these findings to important parameters of the disease. Studying the tear film proteins along with corneal topography modifications as well as with corneal biomechanical parameters is essential in understanding the course of the disease. We have demonstrated that the protein composition of tears is changed in keratoconus by increased levels of proteins with inflammatory properties such as albumin or by decreased levels of proteins with anti-inflammatory properties such as lactoferrin.

Further research is needed to elucidate whether these changes may contribute directly to the development of the disease. 


\author{
Abbreviations \\ AAA - Anti-Albumin Antibody \\ ALDH3 - aldehyde dehydrogenase 3 \\ CCT - central corneal thickness \\ $\mathrm{CH}$ - corneal hysteresis \\ CRF - corneal resistance factor \\ ELISA - enzyme-linked immunosorbent assay \\ Ig A - imunoglobulin A \\ IL-6 - interleukin 6 \\ IL-8 - interleukin 8 \\ IL-a- $\beta$ - interleukin beta \\ $\mathrm{KC}$ - keratoconus \\ $\mathrm{K}$ max - highest corneal value \\ LTF - Lactoferrin \\ LZM - Lysozyme \\ MMP 9- matrix metalloproteinase 9 \\ ROS - reactive oxygen species \\ TNF- $\alpha$ - tumor necrosis factor alpha
}

\section{Acknowledgement}

This work was supported by a grant of the Romanian National Authority for Scientific Research and Innovation CNCS-UEFISCDI (Romania), project code PN-III-P2-2.1-PED-2016-0187.

\section{Authors' Contributions}

MGB (Conception and design; Acquisition of data; Analysis and Interpretation of data; Writing Manuscript; Final approval), MC (Conception and design; Acquisition of data; Analysis and Interpretation of data; Writing Manuscript; Final approval); GI (Conception and design; Acquisition of data; Analysis and Interpretation of data; Writing Manuscript; Final approval), DD (Conception and design; Acquisition of data; Analysis and Interpretation of data; Writing Manuscript; Final approval), CI (Conception and design; Acquisition of data; Analysis and Interpretation of data; Writing Manuscript; Final approval), DS (Conception and design; Acquisition of data; Analysis and Interpretation of data; Writing
Manuscript; Final approval), VP (Conception and design; Acquisition of data; Analysis and Interpretation of data; Writing Manuscript; Final approval), VC (Conception and design; Acquisition of data; Analysis and Interpretation of data; Writing Manuscript; Final approval). All the authors had equally contributed in elaborating the paper.

\section{Conflicts of interest}

The authors declare that there is no conflict of interests regarding the publication of this paper.

\section{References}

1. Gu S, Fan Z, Wang L, Tao X, Zhang Y, Mu G. Corneal Collagen Crosslinking with Hypoosmolar Riboflavin Solution in Keratoconic Corneas. Biomed Res Int. 2014;2014:754182. DOI: 10.1155/2014/754182

2. de Bonis P, Laborante A, Pizzicoli C, Stallone R, Barbano R, Longo $\mathrm{C}$ et al. Mutational screening of VSX1, SPARC,SOD1, LOX, and TIMP3 in keratoconus. Mol Vis. 2011; 17: 2482-94.

3. Gordon-Shaag A, Millodot M, Shneor Liu Y. The genetic and environmental factors for keratoconus. Biomed Res Int. 2015;2015:795738. DOI: 10.1155/2015/795738

4. Mas Tur V, MacGregor C, Jayaswal R, O'Brart D, Maycock N. A review of keratoconus:Diagnosis, pathophysiology, and genetics. Surv Opthalmol.2017;62(6):770-83. DOI: 10.1016/j.survophthal.2017.06.009

5. Paliwal P, Singh A, Tandon R, Titiyal JS, Sharma A. A novel VSX 1 mutation identified in an individual with keratoconus in India. Mol Vis. 2009;15:2475-79.

6. Acera A, Vecino E, Agirretxe IR, Aloria K, Arizmendi JM, Morales C, Duran JA. Changes in tear protein profile in keratoconus disease. Eye. 2011; 25:1225-33. DOI: 10.1038/eye.2011.105

7. Wilson SE, He YG, Weng J, Li Q, Mc Dowall AW, Vital M, Chwang Epithelial injury induces keratocyte apoptosis:hypothesized role for the interleukin-1 system in the modulation of corneal tissue organisation and wound healing. Exp Eye Res. 1996;62:325-27. DOI: 10.1006/exer.1996.0038

8. Lema I, Sobrino T, Duran JA, Brea D, Feijoo-Diez E. 
Subclinical keratoconus and inflammatory molecules from tears.Br J Ophthal. 2009;93(6):820-24. DOI: 10.1136/bjo.2008.144253

9. Nishtala K, Pahuja N, Shetty R, MMA Nuijts R, Ghosh A. Tear biomarkers for keratoconus. Eye Vis. 2016;3(19):1-7 DOI: 10.1186/s40662-016-0051-9

10. de Waal Malefyt R, Abrams J, Bennett B, Figdor CG, de Vries JE. Interleukin 10 inhibits cytokine synthesis by human monocytes: an autoregulatory role of IL-10 produced by monocytes. J Exp Med. 1991;174(5):120920. DOI: $10.1084 /$ jem.174.5.1209

11. Balasubramanian SA, Pye DC, Wilcoxx MDP. Levels of lactoferrin, secretory IgA and serum albumin in the tear of people with keratoconus. Exp Eye Res. 2011;96:132-37. DOI: 10.1016/j.exer.2011.12.010

12. Mazzotta C, Balestrazzi A, Traversi C, Baiocchi S, Caporossi $\mathrm{T}$, Tommasi $\mathrm{C}$ et al. Treatment of progressive keratoconus by riboflavin-UVA-induced crosslinking of corneal colagen:: ultrastructural analysis by Heidelberg Retinal Tomograph II in vivo confocal microscopy in humans. Cornea. 2007;26(4):390-97. DOI: 10.1097/
ICO.0b013e318030df5a

13. Flanagan JL, Willcox MDP. Role of lactoferrin in the tear film. Biochimie. 2009;91(1):35-43. DOI: 10.1016/j.biochi.2008.07.007

14. Lema I, Brea D, Rodriguez-Gonzales R, Diez-Feijoo E, Sobrino T. Proteomic analysis of the tear film in patients with keratoconus. Mol Vis. 2010;16:2055-61.

15. Ohashi Y, Dogru M, Tsubota K. Laboratory findings in tear fluid analysis. Clin Chim Acta. 2006;369(1):17-28. DOI: 10.1016/j.cca.2005.12.035

16. Gorskova EN, Tarasova LN,Teplova SN, Sevostianov EN, Alekhina TV. State of local immunity in keratoconus. 3 Vestnilk Oftalmologii.1998;114(3):26-28.

17. Schnetler R, Gillan WDH, Korseen G. Immunological and antimicrobial molecules in human tears: a review and preliminary report. S Afr Optom.2012;71(3):123-32. DOI: 10.4102 /aveh.v71i3.79

18. Bao F, Geraghty B, Wang Q, Elsheikh A. Consideration of corneal biomechanics in the diagnosis and management of keratoconus:is it important? Eye Vis (Lond). 2016;3:18. DOI: 10.1186/s40662-016-0048-4 